TAIWANESE JOURNAL OF MATHEMATICS

Vol. 1, No. 3, pp. 315-325, September 1997

\title{
ITERATIVE CONSTRUCTION OF FIXED POINTS OF ASYMPTOTIC 1-SET CONTRACTIONS IN BANACH SPACES
}

\author{
P. Vijayaraju
}

\begin{abstract}
We prove theorems on the existence of fixed points and the structure of fixed point sets for asymptotic 1-set contraction mappings $T$ on certain subsets of Banach spaces by assuming some condition on $T$. We also prove some fixed point theorems for a sum of asymptotic 1-set contraction and compact (strongly continuous) mappings in real Banach spaces (reflexive real Banach spaces).
\end{abstract}

\section{INTRODUCTION}

Let $K$ be a nonempty closed convex bounded subset of a Banach space $X$. Sadovskii [9] proved that any condensing self-mapping of $K$ has a fixed point in $K$. This result was extended by Browder [1, Theorem 13.8, p. 230] to a 1-set contraction mapping $T$ by assuming an additional condition that

(i) $(I-T)(K)$ is closed, where I denotes the identity map.

Krasnose1skii [4] proved first that a sum $T+S$ of a contraction mapping $T$ and a compact mapping $S$ with $T x+S y$ in $K$ for all $x, y \in K$, has a fixed point in $K$. This result was extended by Edmunds [2] and Reinermann [8] to a sum of a nonexpansive mapping $T$ (that is, $\|T x-T y\| \leq\|x-y\|$ for all $x, y$ in $K$ ) and a strongly continuous mapping $S$ (that is, $x_{n} \rightarrow x$ in $K$ implies $S x_{n} \rightarrow S x$ as $\left.n \rightarrow \infty\right)$ in Hilbert spaces and in uniformly convex Banach spaces. Singh [10] extended the above results to a sum of such mappings in reflexive Banach spaces by assuming further that $(I-T)(K)$ is demiclosed in the sense that if for any sequence $\left\{x_{n}\right\} \subset K$ which converges weakly to $x \in K$, the convergence of the sequence $\left\{(I-T) x_{n}\right\}$ to $y \in X$ implies that

Received July 1, 1996; revised January 16, 1997.

Communicated by P. Y. Wu.

1991 Mathematics Subject Classification: 47H10, $47 \mathrm{H} 09$.

Key words and phrases: Asymptotic 1-set contraction, fixed point, uniformly asymptotically regular. 
$(I-T) x=y$. A sum $T+S$ of an asymptotic 1-set contraction mapping $T$ of $K$ into $K$ and a strongly continuous mapping $S$ of $K$ into $X$ has a fixed point in $K$, under some additional conditions on $T$ and $T^{n}+S$ for $n=1,2, \cdots$ (when $X$ is a reflexive Banach spaces). This result has been proved recently by the author in [12].

Petryshyn [6] dropped the convexity on the set $K$ of Browder's theorem by assuming the following condition:

(ii) there exists a point $u \in K$ such that if $T x-u=\mu(x-u)$ holds for some $x \in \partial K$, then $\mu \leq 1$,

(when $K$ is a nonempty bounded open subset of a real Banach space $X, \bar{K}$ and $\partial K$ denote the closure and the boundary of $K$, respectively). This result is also true due to Petryshyn [7, Theorem 1] under the weaker condition that (iii) if $\left\{x_{n}\right\}$ is any sequence in $\bar{K}$ such that $(I-T) x_{n} \rightarrow 0$ as $n \rightarrow \infty$, then there exists a point $z \in \bar{K}$ with $(I-T) z=0$

instead of closedness on the set $(I-T)(\bar{K})$. Using this result, Petryshyn $[7$, Theorems 2.2 and 2.3] established fixed point theorems for a sum $T+S$ of a nonexpansive mapping $T$ and a compact mapping $S$ (a strongly continuous mapping) in real Banach spaces by assuming further that $T+S$ satisfies conditions (ii) and (iii) (in uniformly convex real Banach spaces by assuming further that $T+S$ satisfies condition (iii)).

We shall begin by recalling some definitions needed in the sequel.

Definition 1.1. The Kuratowski measure of noncompactness $\alpha(K)$ [cf. 13, p. 492] of a bounded subset $K$ of a metric space $X$ is defined to be the infimum of the set of all $\varepsilon>0$ with the following property:

$\mathrm{K}$ can be covered by finitely many sets, each of whose diameter is $\leq \varepsilon$.

The properties of $\alpha(K)$ are given in [13].

Definition 1.2. Let $K$ be a nonempty subset of a Banach space $X$. If $T$ maps $K$ into $X$, we say that

(a) $T$ is condensing [cf. 13, p. 492] if $T$ is bounded and continuous and $\alpha(T(M))<\alpha(M)$ for all bounded subsets $M$ of $K$ with $\alpha(M)>0$;

(b) $T$ is 1 -set contraction ([10]) if $T$ is bounded and continuous and $\alpha(T(M))$ $\leq \alpha(M)$ for all bounded subsets $M$ of $K$;

(c) $T$ is asymptotic 1-set contraction ([12]) if $T$ is bounded and continuous, and $\alpha\left(T^{n}(M)\right) \leq k_{n} \alpha(M)$ for all bounded subsets $M$ of $K, n=1,2, \cdots$, where $\left\{k_{n}\right\}$ is a sequence of real numbers with $k_{n} \rightarrow 1$ as $n \rightarrow \infty$. 
It is assumed that $k_{n} \geq 1$ and $k_{n} \geq k_{n+1}, n=1,2, \cdots$.

Definition 1.3. Let $K$ and $X$ be as in Definition 1.2. Then the mapping $T$ from $K$ to $X$ is said to be proper ([cf. 13, p. 498]) if the preimage $T^{-1}(M)$ of every compact subset $M$ of $X$ is compact.

A self-mapping $T$ of $K$ is said to be Lipschitzian with Lipschitz constant $\lambda$ if there is a $\lambda \geq 0$ such that

$$
\|T x-T y\| \leq \lambda\|x-y\| \text { for all } x, y \in K .
$$

A mapping $T$ from $K$ to $X$ is called demicompact ([5]) in $K$ if it has the property that, whenever $\left\{x_{n}\right\} \subset K$ is a bounded sequence and $\left\{(I-T) x_{n}\right\}$ is a convergent sequence in $X,\left\{x_{n}\right\}$ converges to a point of $K$.

A mapping $T$ from $K$ to $K$ is said to be uniformly asymptotically regular ([11]) if for each $\eta>0$, there exists $N(\eta)$ (=N, say) such that

$$
\left\|T^{n} x-T^{n+1} x\right\| \leq \eta, \text { whenever } n \geq N, \text { for all } x \in K .
$$

\section{Fixed Points of Asymptotic 1-Set Contraction Mappings}

Theorem 2.1. Let $K$ be a nonempty closed convex bounded subset of a Banach space $X$. Let $T$ be an asymptotic 1-set contraction self-mapping of $K$. Assume further that the following conditions hold:

(a) $\lim _{n \rightarrow \infty}\left[\sup \left\{\left\|T x-T^{n} x\right\|: x \in K\right\}\right]=0$,

(b) $(I-T)(K)$ is closed.

Then $T$ has a fixed point in $K$.

Proof. For fixed $y \in K$, let $T_{n}$ be a mapping of $K$ into itself defined by

$$
T_{n} x=\left(1-a_{n}\right) y+a_{n} T^{n} x \text { for all } x \in K, n=1,2, \cdots,
$$

where $a_{n}=\left(1-\frac{1}{n}\right) / k_{n}$ and $\left\{k_{n}\right\}$ is as in Definition 1.2 (c).

Since $K$ is convex, it follows that $T_{n}$ maps $K$ into itself. Suppose that $M \subset K$ is arbitrary. Then we have

$$
\begin{aligned}
\alpha\left(T_{n}(M)\right) & =\alpha\left(\left(1-a_{n}\right) y+a_{n} T^{n}(M)\right) \leq a_{n} k_{n} \alpha(M) \\
& =\left(1-\frac{1}{n}\right) \alpha(M)(\text { since } T \text { is asymptotic } 1-\text { set contraction }) \\
& <\alpha(M) .
\end{aligned}
$$


Therefore $T_{n}$ is a condensing mapping on $K$.

From Sadovskii's theorem, $T_{n}$ has a fixed point, say, $x_{n}$ in $K$. Therefore $x_{n}-T^{n} x_{n}=\left(1-a_{n}\right)\left(y-T^{n} x_{n}\right) \rightarrow 0$ as $n \rightarrow \infty$, since $a_{n} \rightarrow 1$ as $n \rightarrow \infty$ and $K$ is bounded. By condition (a), we obtain

$$
x_{n}-T x_{n} \rightarrow 0 \text { as } n \rightarrow \infty .
$$

Since $(I-T)(K)$ is closed, $0 \in(I-T)(K)$ and hence there is a point $u$ in $K$ such that $0=(I-T) u$. Thus $u$ is a fixed point of $T$ in $K$.

Remark 2.1. If $K$ is a nonempty weakly compact subset of a Banach space and if $T$ is a mapping of $K$ into itself such that $I-T$ is demiclosed, then $(I-T)(K)$ is closed. Therefore, we obtain the following results.

Corollary 2.1. Let $K$ be a nonempty weakly compact convex subset of a Banach space $X$. Let $T$ be an asymptotic 1-set contraction on $K$ for which the condition (a) of Theorem 2.1 holds. Assume further that (c) $I-T$ is demiclosed. Then $T$ has a fixed point in $K$.

Corollary 2.2. Let $K$ be a nonempty closed convex bounded subset of a reflexive Banach space $X$. Let $T$ be an asymptotic 1 -set contraction on $K$ for which the condition (a) of Theorem 2.1 and the condition (c) of Corollary 2.1 hold. Then $T$ has a fixed point in $K$.

We note that the condition (a) of Theorem 2.1 implies that the map $T$ is uniformly asymptotically regular.

The next theorem is an extension of Theorem 13.8 of Browder [1] to Lipschitzian, asymptotic 1-set contractions which are uniformly asymptotically regular mappings.

Theorem 2.2. Let $K$ be a nonempty closed convex bounded subset of a Banach space $X$. Suppose that $T$ is a Lipschitzian, asymptotic 1-set contraction self-mapping of $K$ with Lipschitz constant $\lambda$. Assume further that $T$ is a uniformly asymptotically regular self-mapping of $K$ such that $(I-T)(K)$ is closed. Then $T$ has a fixed point in $K$.

Proof. Define a map $T_{n}$ from $K$ to $K$ as in the proof of Theorem 2.1. Proceeding as in Theorem 2.1, there is a point $x_{n}$ in $K$ such that

$$
x_{n}-T^{n} x_{n} \rightarrow 0 \text { as } n \rightarrow \infty .
$$


Since $T$ is Lipschitzian, uniformly asymptotically regular, it follows that

$$
\begin{aligned}
\left\|x_{n}-T x_{n}\right\| & \leq\left\|x_{n}-T^{n} x_{n}\right\|+\left\|T^{n} x_{n}-T^{n+1} x_{n}\right\|+\left\|T^{n+1} x_{n}-T x_{n}\right\| \\
& \leq(1+\lambda)\left\|x_{n}-T^{n} x_{n}\right\|+\left\|T^{n} x_{n}-T^{n+1} x_{n}\right\| \\
& \longrightarrow 0 \text { as } n \longrightarrow \infty .
\end{aligned}
$$

Since $(I-T)(K)$ is closed, $0 \in(I-T)(K)$ and hence there is a point $u$ in $K$ such that $u=T u$.

Petryshyn [6] proved the following generalization of Sadovskii's theorem by using a boundary condition (given below) instead of the convexity on the set $K$.

Theorem (A)[6]. Let $K$ be a nonempty open bounded subset of a real Banach space $X$ with $0 \in K$. Suppose that $T$ is a condensing mapping of $\bar{K}$ into $X$ which satisfies the following boundary condition:

(i) If $T x=\mu x$ for some $x \in \partial K$, then $\mu \leq 1$.

Then $T$ has a fixed point in $\bar{K}$.

By using the boundary condition (i) (above), Petryshyn [6] obtained a fixed point theorem for a 1-set contraction of a nonempty open bounded subset of a real Banach space $X$ into $X$. The next theorem is a generalization of this result to Lipschitzian, asymptotic 1-set contractions which are uniformly asymptotically regular maps in such spaces.

Theorem 2.3. Let $K$ be a nonempty open bounded subset of a real Banach space $X$ with $0 \in K$. Suppose that $T$ is a Lipschitzian, asymptotic 1-set contraction self-mapping of $\bar{K}$ with Lipschitz constant $\lambda$, and that it is a uniformly asymptotically regular mapping for which the following conditions hold:

(a) if for each $n=1,2, \cdots, T^{n} y_{n}=\mu_{n} y_{n}$ for some $y_{n} \in \partial K$, then $\mu_{n} \leq 1$.

(b) $(I-T)(\bar{K})$ is closed.

Then $T$ has a fixed point in $\bar{K}$.

Proof. We define a map $T_{n}$ from $\bar{K}$ to $X$ by

$$
T_{n} x=b_{n} T^{n} x \text { for all } x \in \bar{K} \text { and } n=1,2, \cdots,
$$

where $\left\{b_{n}\right\}$ is a sequence of real numbers with $0<b_{n} k_{n}<1$ and $b_{n} \rightarrow 1$ as $n \rightarrow \infty$ and $k_{n}$ is as in Definition $1.2(\mathrm{c})$. Since $T(\bar{K}) \subset \bar{K} \subset X, T_{n}$ maps $\bar{K}$ into $X$. 
Suppose that for each $n=1,2, \cdots, T_{n} y_{n}=\mu_{n} y_{n}$ for some $y_{n}$ in $\partial K$. Then we have $b_{n} T^{n} y_{n}=\mu_{n} y_{n}$ and therefore $T^{n} y_{n}=\left(\mu_{n} / b_{n}\right) y_{n}$. By $(\mathrm{a}), \mu_{n} / b_{n} \leq 1$ and therefore $\mu_{n} \leq b_{n}<1 / k_{n} \leq 1$, since $k_{n} \geq 1$. Thus $T_{n}$ satisfies the condition (i) of Theorem (A).

Suppose that $M \subset \bar{K}$ is arbitrary. Then we have

$$
\begin{aligned}
\alpha\left(T_{n}(M)\right)= & b_{n} \alpha\left(T^{n}(M)\right) \leq b_{n} k_{n} \alpha(M) \\
& (\text { since } T \text { is an asymptotic } 1-\text { set contraction on } \bar{K}) \\
< & \alpha(M), \text { since } 0<b_{n} k_{n}<1 .
\end{aligned}
$$

Therefore $T_{n}$ is a condensing mapping of $\bar{K}$ into $X$. From Theorem (A), $T_{n}$ has a fixed point, say, $x_{n}$ in $\bar{K}$. The remaining part of the proof is similar to that of Theorem 2.2.

Theorem 2.4. Let $K$ and $X$ be as in Theorem 2.3. If $T$ is a demicompact, Lipschitzian and asymptotic 1-set contraction mapping of $\bar{K}$ into itself with Lipschitz constant $\lambda$ which is a uniformly asymptotically regular map for which the condition (a) of Theorem 2.3 holds, then the set $F(T)$ of fixed points of $T$ is nonempty and compact.

Proof. Since $T$ is demicompact and continuous, it follows that $(I-T)(\bar{K})$ is closed. From Theorem 2.3, $F(T) \neq \emptyset$. Suppose that $\left\{x_{n}\right\}$ is any sequence in $F(T)$. Since $T$ is demicompact in $\bar{K}$, there is a subsequence $\left\{x_{n_{k}}\right\}$ of $\left\{x_{n}\right\}$ such that

$$
x_{n_{k}} \rightarrow x \in \bar{K} \text { as } k \rightarrow \infty \text {. }
$$

Since $I-T$ is continuous, $(I-T) x_{n_{k}} \rightarrow(I-T) x$ as $k \rightarrow \infty$. Therefore $(I-T) x=0$. Hence $x \in F(T)$. Thus $\left\{x_{n}\right\}$ has a subsequence $\left\{x_{n_{k}}\right\}$ which is convergent to $x$ in $F(T)$. This means that $F(T)$ is compact.

If $0 \notin K$ in Theorem 2.3, we obtain the following result.

Theorem 2.5. Let $K$ be a nonempty open bounded subset of a real Banach space $X$. Suppose that $T$ is a Lipschitzian, asymptotic 1-set contraction mapping of $\bar{K}$ into itself with Lipschitz constant $\lambda$ which satisfies the following conditions:

$\left(\mathrm{a}_{1}\right)$ there exists $u$ in $K$ such that if for each $n=1,2, \cdots, T^{n} y_{n}-u=\mu_{n}\left(y_{n}-\right.$ u) for some $y_{n}$ in $\partial K$, then $\mu_{n} \leq 1$.

(b) $(I-T)(\bar{K})$ is closed. 
Assume further that $T$ is a uniformly asymptotically regular self-mapping of $\bar{K}$. Then $T$ has a fixed point in $\bar{K}$.

Proof. Suppose that $A=K-u=\{x-u: x \in K\}$. Then $A \neq \phi$ as $0 \in A$. Since $K$ is open and bounded, so is $A$. Also $\partial A=\partial K-u$ and $\bar{A}=\bar{K}-u$.

We define a map $S$ from $\bar{A}$ to $\bar{A}$ by

$$
S(x-u)=T x-u \text { for all } x-u \in \bar{A} .
$$

Since $T$ maps $\bar{K}$ into itself, $S$ maps $\bar{A}$ into itself. Suppose that $M \subset \bar{A}$ is arbitrary. Then we have

$$
\alpha\left(S^{n}(M)\right)=\alpha\left(T^{n}(M+u)-u\right)=\alpha\left(T^{n}(M+u)\right) \leq k_{n} \alpha(M+u)=k_{n} \alpha(M),
$$

since $T$ is an asymptotic 1 -set contraction on $\bar{K}$. Therefore $S$ is an asymptotic 1-set contraction on $\bar{A}$. Since $T$ is Lipschitzian, uniformly asymptotically regular, so is $S$. Now, let for each $n=1,2, \cdots, S^{n} z_{n}=\mu_{n} z_{n}$ for some $z_{n}=y_{n}-u$ in $\partial A$, where $y_{n}$ in $\partial K$. Then $T^{n} y_{n}-u=\mu_{n}\left(y_{n}-u\right)$ and by $\left(a_{1}\right), \mu_{n} \leq 1$.

Therefore the condition (a) of Theorem 2.3 holds for $S$. Since $(I-S)(x-$ $u)=(I-T) x$ for all $x$ in $\bar{K}$, it follows that $(I-S)(\bar{A})=(I-T)(\bar{K})$, and hence $(I-S)(\bar{A})$ is closed. Thus $A$ and $S$ satisfy all the hypotheses of Theorem 2.3. Therefore there is a point $y=x-u$ in $\bar{A}$ such that $S y=y$ that is, $S(x-u)=x-u$ and therefore $T x=x$. This means that $T$ has a fixed point $x$ in $\bar{K}$.

Theorem 2.6. Let $K$ and $X$ be as in Theorem 2.5. If $T$ is a demicompact, Lipschitzian and asymptotic 1 -set contraction self-mapping of $\bar{K}$ with Lipschitz constant $\lambda$ and it is a uniformly asymptotically regular map for which the condition $\left(a_{1}\right)$ of Theorem 2.5 holds, then the set $F(T)$ of fixed points of $T$ is nonempty and compact.

Proof. Define A and $\mathrm{S}$ as in the proof of the above theorem. Since $T$ is demicompact and continuous, $\mathrm{S}$ is demicompact and continuous. Therefore $(I-S)(\bar{A})$ is closed. From Theorem 2.5, $F(T) \neq \emptyset$. Since $S$ is demicompact, $F(S)$ is compact and therefore $F(T)$ is compact.

The following results are used to prove our Theorem 2.7.

Theorem (B) ([6]). Let $K$ be a nonempty open bounded subset of a real Banach space $X$. Suppose that $T$ is a 1-set contraction mapping of $\bar{K}$ into $X$ for which the following hold: 
(i) there is a point $u \in K$ such that if $T x-u=\mu(x-u)$ for some $x \in \partial K$, then $\mu \leq 1$.

(ii) $(I-T)(\bar{K})$ is closed.

Then $T$ has a fixed point in $\bar{K}$.

Theorem (C)([13, p. 498]). Suppose that $K$ is a nonempty closed bounded subset of a Banach space $X$. If $T$ is a condensing mapping of $K$ into $X$, then the map $I-T$ is proper on $K$.

Theorem (D)([13, p. 499]). Suppose that $K$ is a nonempty closed subset of a Banach space $X$. If $T$ is a continuous and proper mapping of $K$ into $X$, then the set $T(K)$ is closed.

The following theorem shows that if the closedness of the set $(I-T)(\bar{K})$ in Theorem 2.5 is replaced by the condition $\left(b_{1}\right)$ below, then the conclusion of this result remains valid. This result is an extension of Theorem 1 of Petryshyn [7] for 1-set contraction mappings.

Theorem 2.7. Let $K$ be a nonempty open bounded subset of a real Banach space $X$. Suppose that $T$ is a Lipschitzian, asymptotic 1-set contraction selfmapping of $\bar{K}$ with Lipschitz constant $\lambda$ which satisfies the condition $\left(a_{1}\right)$ of Theorem 2.5. Assume further that $T$ is a uniformly asymptotically regular self-mapping of $\bar{K}$ and $T$ satisfies the following condition:

$\left(b_{1}\right)$ if $\left\{x_{n}\right\}$ is any sequence in $\bar{K}$ such that $x_{n}-T x_{n} \rightarrow 0$ as $n \rightarrow \infty$, then there exists $z \in \bar{K}$ with $(I-T) z=0$.

Then $T$ has a fixed point in $\bar{K}$.

Proof. Let $u \in K$ be fixed. Define a map $T_{n}$ from $\bar{K}$ to $X$ by

$$
T_{n} x=\left(1-a_{n}\right) u+a_{n} T^{n} x \text { for all } x \in \bar{K}, n=1,2, \cdots,
$$

where $a_{n}$ is as in Theorem 2.1. Since $T$ is an asymptotic 1-set contraction on $\bar{K}$, it follows that $T_{n}$ is a condensing mapping of $\bar{K}$ into $X$ and hence a 1-set contraction. By Theorem (C), $I-T_{n}$ is proper on $\bar{K}$. By Theorem (D), $\left(I-T_{n}\right)(K)$ is closed. Suppose that for each $n=1,2,3, \cdots, T_{n} y_{n}-u=$ $\mu_{n}\left(y_{n}-u\right)$ for some $y_{n} \in \partial K$. Then we have $a_{n} T^{n} y_{n}+\left(1-a_{n}\right) u-u=\mu_{n}\left(y_{n}-u\right)$, that is, $T^{n} y_{n}-u=\left(\mu_{n} / a_{n}\right)\left(y_{n}-u\right)$. By $\left(a_{1}\right), \mu_{n} / a_{n} \leq 1$ and therefore $\mu_{n} \leq a_{n}<1$. Hence $T_{n}$ satisfies the condition (i) of Theorem (B). Thus $K$ and $T_{n}$ satisfy all conditions of Theorem (B). Therefore there is a point $x_{n}$ in $\bar{K}$ such that $T_{n} x_{n}=x_{n}$. Hence $x_{n}-T^{n} x_{n} \rightarrow 0$ as $n \rightarrow \infty$. 
Since $T$ is Lipschitzian and uniformly asymptotically regular, it follows that

$$
x_{n}-T x_{n} \rightarrow 0 \text { as } n \rightarrow \infty .
$$

Therefore, by $\left(b_{1}\right), T$ has a fixed point in $\bar{K}$.

Remark 2.2. If we assume $(I-T)(\bar{K})$ to be closed, then condition $\left(b_{1}\right)$ of the above theorem holds.

\section{Fixed Points for a Sum of Two Mappings}

Using Theorem 2.7, we prove fixed point theorems for a sum of two mappings. These results generalize Theorems 2.2 and 2.3 of Petryshyn [7] for a sum of nonexpansive and compact (strongly continuous) mappings in real Banach spaces (in uniformly convex real Banach spaces).

Theorem 3.1. Let $K$ be a nonempty open bounded subset of a real Banach space $X$. Suppose that $T$ is an asymptotic 1-set contraction on $\bar{K}$ and $S$ is a compact self-mapping of $\bar{K}$. Suppose that $T+S$ is a Lipschitzian, uniformly asymptotically regular mapping of $\bar{K}$ into itself and satisfies the conditions $\left(a_{1}\right)$ of Theorem 2.5 and $\left(b_{1}\right)$ of Theorem 2.7 with $T+S$ in place of $T$. Then $T+S$ has a fixed point in $\bar{K}$.

Proof. Since $T$ is an asymptotic 1-set contraction and $S$ is compact, it follows from Theorem 2.2 of [12] that $T+S$ is an asymptotic 1-set contraction in $\bar{K}$ and hence the proof of this theorem follows from that of Theorem 2.7.

Theorem 3.2. Let $K$ be a nonempty open bounded subset of a reflexive real Banach, space $X$. Suppose that $T$ is an asymptotic 1-set contraction on $\bar{K}$ such that $I-T$ is demiclosed and $S$ is a strongly continuous self-mapping of $\bar{K}$. Suppose that $T+S$ is a Lipschitzian, uniformly asymptotically regular mapping of $\bar{K}$ into itself and satisfies the condition $\left(a_{1}\right)$ of Theorem 2.5 with $T+S$ in place of $T$. Then $T+S$ has a fixed point in $\bar{K}$.

Proof. Since $X$ is reflexive and $K$ is bounded, every sequence $\left\{x_{n}\right\}$ in $K$ has a weakly convergent subsequence $\left\{x_{n_{k}}\right\}$,

that is, $x_{n_{k}} \rightarrow x$ as $k \rightarrow \infty$ for some $x$ in $K$.

Since $S$ is strongly continuous, $S x_{n_{k}} \rightarrow S x$ as $k \rightarrow \infty$. Therefore $S$ is compact.

Since $T$ is an asymptotic 1-set contraction and $S$ is compact, it follows from Theorem 2.2 of [12] that $T+S$ is an asymptotic 1-set contraction on $\bar{K}$. Suppose that $\left\{x_{n}\right\}$ is any sequence in $\bar{K}$ such that

$$
x_{n}-(T+S) x_{n} \rightarrow 0 \text { as } n \rightarrow \infty .
$$


From Lemma 2.1 of [12], $(I-T-S)(\bar{K})$ is closed. Therefore $0 \in(I-T-S)(\bar{K})$ and hence there is a point $z \in \bar{K}$ such that $z-(T+S) z=0$. Thus condition $\left(b_{1}\right)$ of Theorem 2.7 is satisfied. Therefore the conclusion of this theorem follows from Theorem 2.7.

\section{ACKNOWLEDGEMENT}

The author would like to thank Professor T. R. Dhanapalan for proposing this problem and for his many helpful discussions during the work on this paper. The author wishes to thank the referee for suggestions that lead to a better presentation of this paper.

\section{REFERENCES}

1. F. E. Browder, Nonlinear Operators and Nonlinear Equations of Evolution in Banach Spaces, Proc. Sympos. Pure Math., Vol. 18, Part 2, Amer. Math. Soc., Providence, R. I., 1976.

2. D. E. Edmunds, Remarks on nonlinear functional equation, Math. Ann. 174 (1967), 233-239.

3. K. Goebel and W. A. Kirk, A fixed point theorem for asymptotically nonexpansive mappings, Proc. Amer. Math. Soc. 35 (1972), 171-174.

4. M. A. Krasnoselskii, Two remarks on the method of successive approximations, Uspekhi Mat. Nauk 10 (1955), 123-127. (in Russian)

5. W. V. Petryshyn, Construction of fixed points of demicompact mappings in Hilbert space, J. Math. Anal. Appl. 14 (1966), 276-284.

6. W. V. Petryshyn, Structure of the fixed points sets of k-set-contractions, Arch. Rational Mech. Anal. 40 (1971), 312-328.

7. W. V. Petryshyn, Fixed point theorems for various classes of 1-set-contractive and 1-ball-contractive mappings in Banach spaces, Trans. Amer. Math. Soc. 182 (1973), 323-352.

8. J. Reinermann, Fixpunktsätze vom Krasnoselski-Typ, Math. Z. 119 (1971), 339-334.

9. B. N. Sadovskii, On a fixed point theorem, Funktsional. Anal. i Prilozhen. 1 (1967), 74-76.

10. S. P. Singh, Fixed point theorems for a sum of nonlinear operators, Rend. Accad. Naz. Lincei 54 (1973), 1-4.

11. P. Vijayaraju, Fixed point theorems for asymptotically nonexpansive mappings, Bull. Calcutta Math. Soc. 80 (1988), 133-136. 
12. P. Vijayaraju, A fixed point theorem for a sum of two mappings in reflexive Banach spaces, Math. J. Toyoma Univ. 14 (1991), 41-50.

13. E. Zeidler, Nonlinear Functional Analysis and its Applications, Vol. I, Fixed Point Theorems, Springer Verlag, New York, Berlin, Heidelberg, Tokyo, 1986.

Department of Mathematics, Anna University,

Madras - 600 025, India.

E-mail: annalib@sirnetm.ernet.in 